\title{
Inhibitory cueing effects following manual and saccadic responses to arrow cues
}

\author{
Yun Ding ${ }^{1,2} \cdot$ Tao He $^{1,2} \cdot$ Jason Satel $^{3} \cdot$ Zhiguo Wang ${ }^{1,2,4}$
}

Published online: 8 March 2016

(C) The Psychonomic Society, Inc. 2016

\begin{abstract}
With two cueing tasks, in the present study we examined output-based inhibitory cueing effects (ICEs) with manual responses to arrow targets following manual or saccadic responses to arrow cues. In all experiments, ICEs were observed when manual localization responses were required to both the cues and targets, but only when the cue-target onset asynchrony (CTOA) was 2,000 ms or longer. In contrast, when saccadic responses were made in response to the cues, ICEs were only observed with CTOAs of 2,000 ms or less - and only when an auditory cue-back signal was used. The present study also showed that the magnitude of ICEs following saccadic responses to arrow cues decreased with time, much like traditional inhibition-of-return effects. The magnitude of ICEs following manual responses to arrow cues, however, appeared later in time and had no sign of decreasing even $3 \mathrm{~s}$ after cue onset. These findings suggest that ICEs linked to skeletomotor activation do exist and that the ICEs evoked by oculomotor activation can carry over to the skeletomotor system.
\end{abstract}

Zhiguo Wang

z.wang@hznu.edu.cn

1 Department of Psychology, Hangzhou Normal University, Hangzhou, China

2 Zhejiang Key Laboratory for Brain and Cognitive Disorder Assessment Research, Hangzhou, China

3 School of Psychology, University of Nottingham Malaysia Campus, Semenyih, Malaysia

4 Department of Cognitive Science, Macquarie University, Sydney, New South Wales, Australia
Keywords Spatial attention · Inhibition of return · Eye movements $\cdot$ Cueing paradigm $\cdot$ Inhibitory cueing effects

A peripheral onset cue can briefly capture attention and facilitate responses to targets at the same location (Jonides, 1981). Later on, however, an inhibitory cueing effect (ICE) emerges at the cued location and delays target responses (Berlucchi, Di Stefano, Marzi, Morelli, \& Tassinari, 1981; Cohen, 1981; Posner \& Cohen, 1984). This later ICE was named "inhibition of return" (IOR) by Posner, Rafal, Choate, and Vaughan (1985), to reflect the theoretical proposition that, once attention has left a location, it is inhibited to return. As a theoretical construct implied in Posner et al. (1985), IOR entails both a cause and an effect: "In cause, IOR occurs in the aftermath of oculomotor activation" and "in effect, IOR is a long-lasting response bias that affects overt and covert orienting" (Hilchey, Klein, \& Satel, 2014, p. 1604). For clarity, in the present article we will use the term ICE rather than IOR to describe cueing effects that may have been caused by IOR or, alternatively, by other mechanisms functionally similar to it.

Posner and Cohen (1984) originally suggested that the ICE evoked by peripheral cues was the result of sensory stimulation (i.e., an input-based effect). Posner et al. (1985), however, found that this ICE had no effect on the perceptual arrival time of targets (see also Klein, Schmidt, \& Müller, 1998; Maylor, 1985) but, nevertheless, biased eye movements away (see also Clohessy, Posner, Rothbart, \& Vecera, 1991). These findings suggest that the observed ICE may actually represent a motor bias against previously attended locations (i.e., an outputbased effect). Subsequent studies demonstrated that many ICEs are closely linked with the oculomotor system (e.g., Dorris, Klein, Everling, \& Munoz, 2002; Posner et al., 1985; Rafal, Calabresi, Brennan, \& Sciolto, 1989; Sumner, Nachev, Vora, Husain, \& Kennard, 2004) and can be readily 
evoked in cueing tasks that require eye movement responses (e.g., Chica, Taylor, Lupiáñez, \& Klein, 2010; Klein \& Hilchey, 2011).

Although efficient orienting of the eyes is crucial for visual selection, the skeletomotor system is what enables an individual to actually interact with objects in the external world. Briand, Larrison, and Sereno (2000) found that ICEs appeared later for manual than for saccadic responses (but see Khatoon, Briand, \& Sereno, 2002). With S-cone stimuli that were invisible to the superior colliculus (at least along the retinotectal pathway), Sumner and colleagues (Sumner, 2006; Sumner et al., 2004) observed ICEs with manual but not with saccadic responses. Zhang and Zhang (2011) also found that loading up visual working memory interfered with ICEs measured with manual but not with saccadic responses. In addition, it has been shown that ICEs interact with the Simon effect (Ivanoff, Klein, \& Lupiáñez, 2002) and that ICEs double in magnitude when a nonresponding hand is placed on the keyboard (Ivanoff \& Klein, 2001). All of these findings suggest that the skeletomotor system may also play an important role in the generation and expression of ICEs. However, the use of peripheral onset cues makes it difficult to determine whether these ICEs were also contributed to by a sensory deficit at the cued location (e.g., Fecteau \& Munoz, 2005; Müller \& Kleinschmidt, 2007; Satel, Wang, Trappenberg, \& Klein, 2011). With central arrow cues and targets, Cowper-Smith, Eskes, and Westwood (2013) observed slower reaching responses toward previously touched locations, providing clear evidence that skeletomotor activation also gives rise to outputbased ICEs. Although such an ICE does not meet the theoretical definition of IOR (Hilchey et al., 2014; Posner et al., 1985), it dovetails with the observation of inhibitory tags in manual foraging (Thomas et al., 2006) and affords the function of biasing orienting toward novelty.

Manual reaching responses were required in CowperSmith et al.'s (2013) study. With manual buttonpresses, however, Taylor and Klein (2000) observed no ICE in a similar experimental setup. Since Fischer, Pratt, and Neggers (2003) also failed to observe an ICE when participants made reaching responses to central arrow targets, it seems unlikely that response modes were behind these conflicting findings. One other prominent methodological difference between these two studies was that the cue-target onset asynchrony (CTOA) was only 1,000 ms in Taylor and Klein's study, whereas that in Cowper-Smith et al.'s was 2,800 ms. With central arrow targets, discrimination of the arrow direction is needed before a response can be issued. It is known that ICEs measured with discrimination responses take longer to emerge on a behavioral level (e.g., Lupiáñez, Milán, Tornay, Madrid, $\&$ Tudela, 1997). The primary purpose of the present study was to clarify whether Taylor and Klein's (2000) failure to observe ICEs with manual button responses was due to their relatively short CTOA. It is critical to clear up this issue, because if ICEs can be evoked by skeletomotor activation, they should be observed when the skeletomotor system is actively engaged by either manual reaches (as in Cowper-Smith et al., 2013) or buttonpresses (as in Taylor \& Klein, 2000).

As has been alluded to before, ICEs linked to oculomotor activation have been frequently reported in the literature (e.g., Abrams \& Dobkin, 1994; Posner et al., 1985; Satel, Hilchey, Wang, Story, \& Klein, 2013; Satel \& Wang, 2012; Taylor \& Klein, 2000; Wang, Satel, \& Klein, 2012). Nevertheless, it remains unclear whether these oculomotor ICEs carry over to the skeletomotor system. With saccadic responses to the cue and manual responses to the target, Taylor and Klein (2000) observed ICEs of the same magnitude, regardless of whether the cue and target were central arrows or peripheral onsets. Fischer et al. (2003), however, observed ICEs with saccadic responses, but not with any type of manual response to central arrow targets, and they concluded that "motor-based IOR is restricted to the oculomotor system" (p. 379). This is quite surprising, given that neural activation in the oculomotor system certainly does carry over to the skeletomotor system (e.g., Werner, 1993). A secondary purpose of the present study was to reexamine this issue. Saccadic responses to central arrow cues have been shown to evoke an oculomotor ICEthat is, an ICE that satisfies the theoretical definition of IOR (Hilchey et al., 2014). If oculomotor ICEs do carry over to the skeletomotor system, a robust ICE would also be revealed with manual button responses to central arrow targets.

To achieve these goals, for the present study we adopted two cueing tasks. Manual button responses were required to central arrow targets in both tasks, whereas saccadic responses were required to central arrow cues in one task (saccadicmanual), and manual localization responses were required in the other (manual-manual). On the basis of previous results, we expected saccadic responses to the cues to evoke an output-based ICE in the oculomotor system (e.g., Chica et al., 2010; Satel \& Wang, 2012; Taylor \& Klein, 2000; Wang et al., 2012). Manual button responses to the cues might also evoke an ICE, but the results of Taylor and Klein (2000) suggest otherwise. In four experiments, ICEs were consistently observed with manual button responses to the cue-but only when the CTOA was relatively long (2,000 ms or longer). In addition, in these experiments we also examined the time courses of ICEs evoked by skeletomotor and oculomotor activation (Exp. 4) and the boundary conditions under which oculomotor ICEs carry over to the skeletomotor system (Exps. 1-3).

\section{Experiment 1: visual cue-back signal}

To examine ICEs evoked by manual and saccadic responses to arrow cues, we first replicated two of Taylor and Klein's 
(2000) 24 cueing tasks, with the addition of a much longer CTOA. Nonpredictive central arrows were used as the cues, and manual localization responses were required to central arrow targets in both tasks. These two tasks were blockedsaccadic responses were made to the cues in the first task (saccadic-manual), whereas manual responses were made to the cues in the second task (manual-manual).

\section{Method}

Participants In all experiments reported here, all participants reported normal or corrected-to-normal visual acuity and had no motor, or neurological abnormalities. They were paid 40 Yuan per hour for their participation. Twenty-three volunteers participated in Experiment 1. One of these was excluded from the analysis because she did not finish the tasks. The mean age of the remaining 22 participants ( 17 females, five males) was 20.82 years.

Apparatus and stimuli The stimuli were presented on a 17-in. CRT monitor, and the viewing distance was maintained at about $62 \mathrm{~cm}$ with a chinrest. Stimulus presentation and response registration were controlled with scripts written in Python. Eye movements were monitored with an EyeLink 1000 (SR Research) eyetracking system. The spatial resolution of the eyetracker was $0.2^{\circ}$ or better, and the sampling rate was set to $500 \mathrm{~Hz}$.

The stimuli were similar to those of Taylor and Klein (2000). Three gray placeholder boxes subtending $1.8^{\circ}$ (visual angle) and were visible at all times. The center-to-center distance between two adjacent boxes was $9^{\circ}$. Both the cue and target stimuli were arrows presented in the central box, measuring $0.8^{\circ}$ (width) by $0.2^{\circ}$ (height).

Design and procedure The sequence of events in both tasks is illustrated in Fig. 1. Self-paced drift correction was performed at the beginning of each trial, with successful drift correction signaled by a beep. Then a fixation cross appeared in the central box, and an error message was displayed if eye movements were detected during a 500-ms fixation period. The cue was an arrow presented for $300 \mathrm{~ms}$ in the central box, pointing either left or right. In the manual-manual task, participants maintained fixation throughout a trial and pressed the "Z" and "/" keys in response to left- and rightpointing arrow cues, respectively. Failure to respond within $500 \mathrm{~ms}$, pressing the wrong key, or making an eye movement triggered an error message and the termination of the trial. In the saccadic-manual task, participants made saccades to the peripheral box indicated by the central arrow. If participants failed to initiate an eye movement within $500 \mathrm{~ms}$, or if the eyes missed the center of the appropriate peripheral box by more than $2.5^{\circ}$, an error message was displayed and the trial was terminated. Five hundred milliseconds after cue onset, the central box was brightened for $300 \mathrm{~ms}$ (visual cueback signal). Participants maintained fixation in the manual-manual task, whereas they made saccades back to the central box in the saccadic-manual task. Failure to maintain fixation in the manual-manual task, or failure to saccade back to the central box within $500 \mathrm{~ms}$, would trigger an error message and the termination of the trial. Unlike Taylor and Klein (2000), who only tested a CTOA of $1,000 \mathrm{~ms}$, in the present experiment we also tested a much longer CTOA $(2,000 \mathrm{~ms})$. These two CTOAs were intermixed within blocks of trials. So, 1,000 or $2,000 \mathrm{~ms}$ following cue onset, an arrow target appeared in the central box and participants quickly responded with the " $Z$ " or the "/" key. An error message was displayed if saccades were detected before the target response or if the participant pressed the wrong key.

In the present experiment, we adopted a 2 (Task: manual-manual vs. saccadic-manual) $\times 2$ (CTOA: 1 , 000 vs. $2,000 \mathrm{~ms}) \times 2$ (Cueing: cued vs. uncued) within-subjects design. Each experimental cell was tested for 32 trials, and thus each participant needed to successfully complete a total of 256 trials. The manual-manual and saccadic-manual tasks were blocked and counterbalanced across participants. Whenever an error message was displayed, the trial was discarded and later presented to the participants in a random order, until all trials had been completed successfully. The participants could take a break after every 80 trials, and a practice

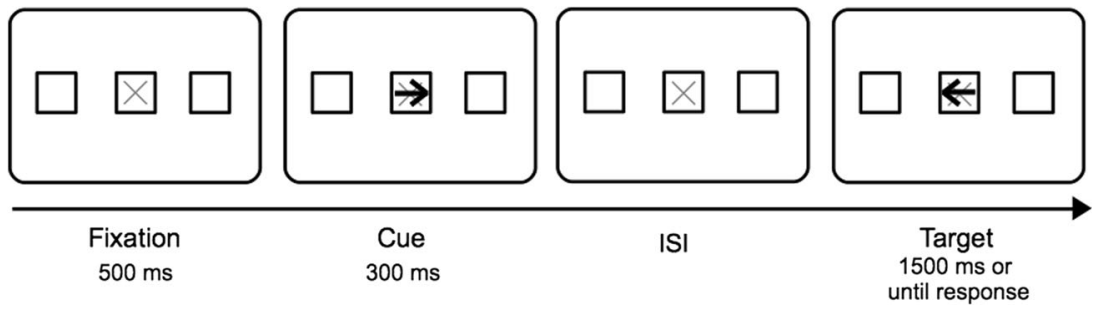

Fig. 1 The display sequence in a sample trial. Participants always made manual localization responses to the target, but also made manual (in the manual-manual task) or saccadic (in the saccadic-manual task) responses to the cue. For illustration purposes, the stimuli are not drawn to scale 
block of eight trials was provided at the beginning of each task.

\section{Results}

All statistical analyses were performed in R (R Development Core Team, 2015). The effect size measure reported for analyses of variance (ANOVAs) was generalized eta squared $\left(\eta_{G}{ }^{2}\right.$; small size $=.02$, medium size $=.13$, and large size $=.26$; see Bakeman, 2005).

Target RTs Only successfully completed trials were considered in the response time (RT) analysis. These RTs were cleansed on a per-participant, per-experimental-cell basis, using the nonrecursive method recommended by Van Selst and Jolicœur (1994). After outlier removal, $97.78 \%$ and $97.22 \%$ of the trials remained in the manual-manual and saccadic-manual tasks, respectively.

The mean target RTs in all conditions are presented in Table 1. A repeated measures ANOVA was performed, revealing significant main effects for task $[F(1,21)=14.71, M S E=$ $\left.3,749, p<.001, \eta_{G}^{2}=.11\right]$ and CTOA $[F(1,21)=17.97, M S E$ $\left.=954, p<.001, \eta_{G}{ }^{2}=.04\right]$. RTs were generally longer in the saccadic-manual task, and were longer in the short-CTOA conditions. The main effect of cueing did not reach significance $\left[F(1,21)=2.80, M S E=1,014, p=.11, \eta_{G}{ }^{2}=.01\right]$, but a two-way interaction between cueing and CTOA was observed $\left[F(1,21)=20.07, M S E=96.6, p<.001, \eta_{G}^{2}=.005\right]$, suggesting that the magnitudes of ICEs differed across CTOAs. The two-way interaction between CTOA and task approached significance $\left[F(1,21)=3.39, M S E=553.9, p=.08, \eta_{G}{ }^{2}=\right.$ $.001]$, whereas that between cueing and task did not reach significance $\left[F(1,21)=1.55, M S E=443.4, p=.23, \eta_{G}^{2}=\right.$ $.002]$. The three-way interaction between task, cueing, and CTOA was not significant $[F(1,21)=2.13, M S E=130.2, p$ $\left.=.16, \eta_{G}^{2}=.001\right]$.

Taylor and Klein's (2000) empirical findings and their theory predicted no ICE in the manual-manual task and a robust ICE in the saccadic-manual task. Thus, planned comparisons were performed to evaluate the ICEs in all conditions, even though the three-way interaction did not reach significance. A reliable ICE $(21 \mathrm{~ms})$ was observed in the manual-manual task when the CTOA was $2,000 \mathrm{~ms}$ $[t(21)=4.06, p<.001]$, but the ICEs in all other conditions did not reach significance [all $t \mathrm{~s}<1.08$, all $p \mathrm{~s}>$.29] (see Fig. 2a).

Target response errors Trials with erroneous eye movements or responses were aborted and recycled. Most recycled trials were terminated before target presentation; only those recycled due to incorrect target responses were considered in the response error analysis. Target response errors are presented in Table 1. Analyses revealed a

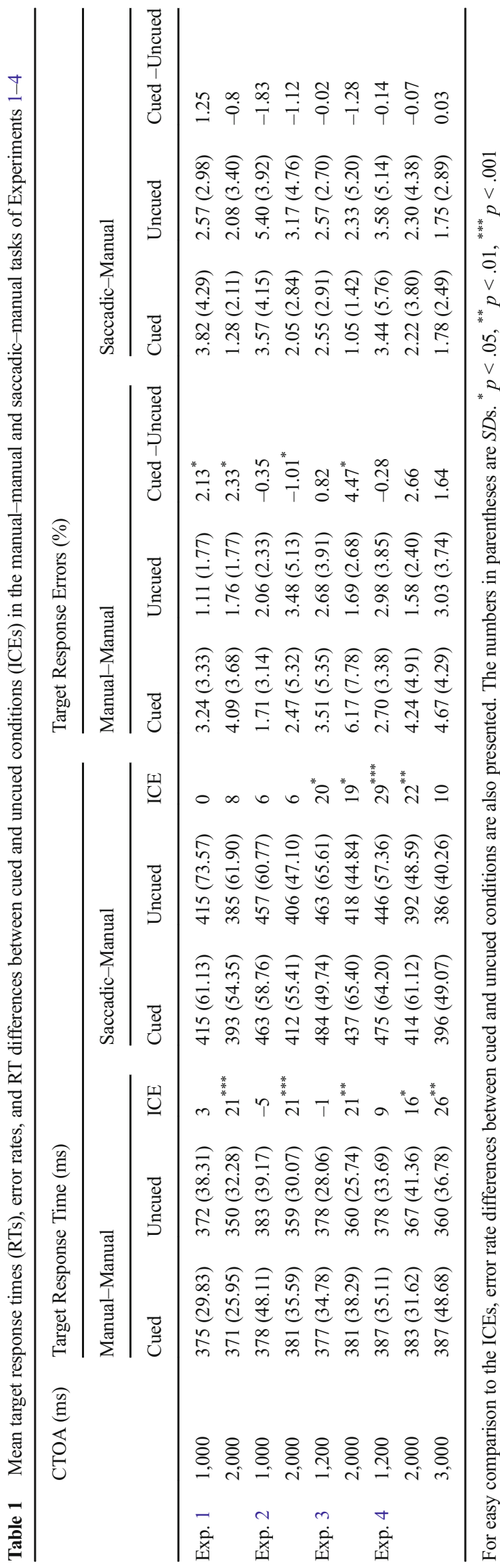



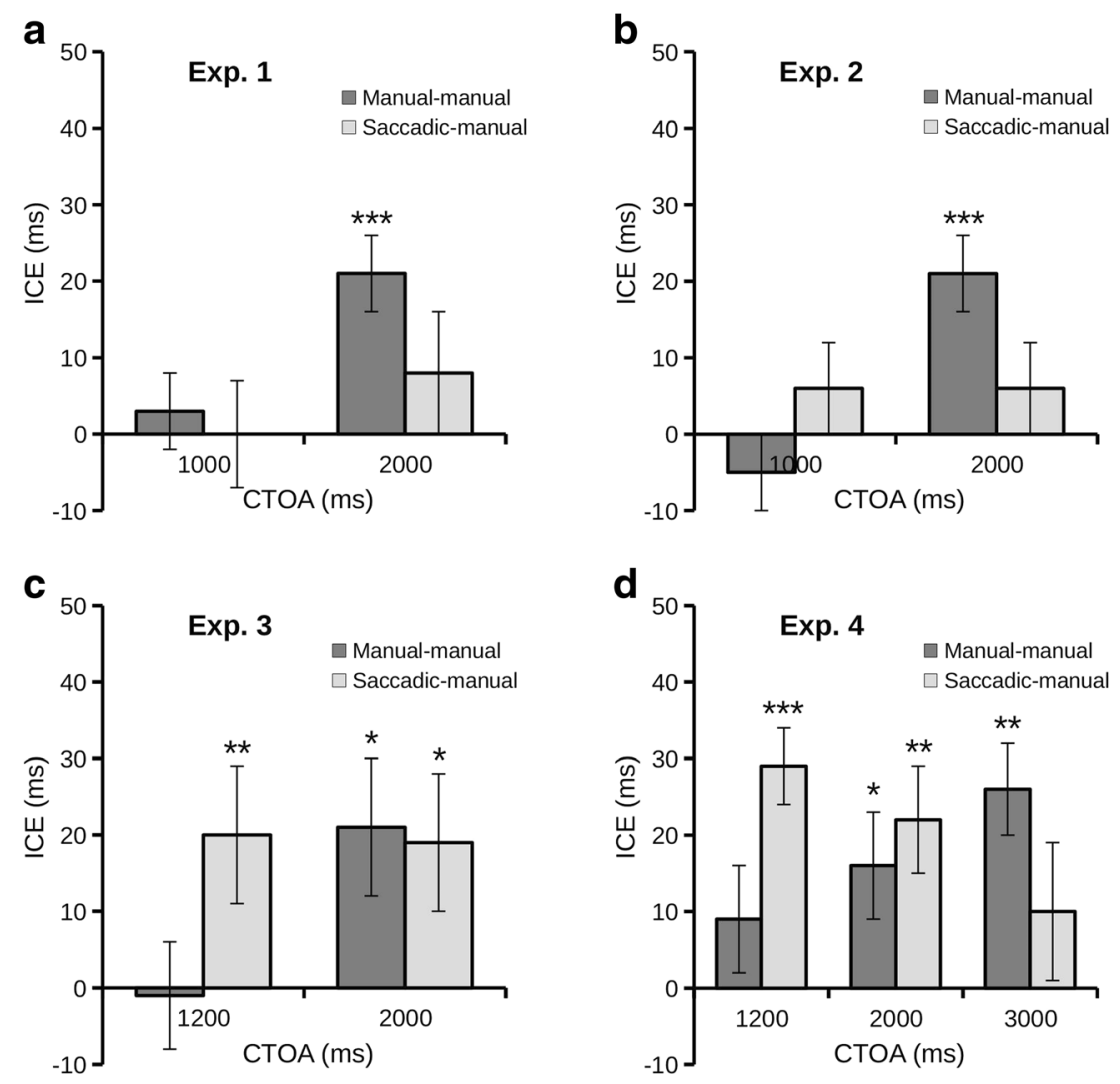

Fig. 2 Inhibitory cueing effects (ICEs) observed in all conditions of (a) Experiment 1 (visual cue-back), (b) Experiment 2 (no cue-back), (c) Experiment 3 (auditory cue-back), and (d) Experiment 4 (time course). Error bars denote $\pm 1 S E M .{ }^{*} p<.05,{ }^{* * *} p<.01,{ }^{* * *} p<.001$

marginal main effect for cueing $[F(1,21)=3.80, M S E=$ $\left.17.47, p=.06, \eta_{G}^{2}=.04\right]$, with higher error rates observed in the cued conditions. A significant two-way interaction emerged between task and CTOA $[F(1,21)=$ 15.86, $\left.M S E=3.58, p<.001, \eta_{G}^{2}=.04\right]$, because the error rate decreased as the CTOA increased in the saccadic-manual task. The two-way interaction between cueing and task was marginally significant $[F(1,21)=4.09$, MSE $\left.=10.78, p=.06, \eta_{G}^{2}=.03\right]$; the error rate was higher in the cued condition, but only in the manual-manual task. All other effects did not reach significance [all $F_{\mathrm{s}}<2.64$, all $p \mathrm{~s}>.11]$.

\section{Discussion}

In the manual-manual task, an ICE was observed for the long, but not the short, CTOA, suggesting that their relatively short CTOA $(1,000 \mathrm{~ms})$ might be the reason for Taylor and Klein's (2000) failure to observe an ICE in this task. Although Taylor and Klein (2000) observed reliable ICEs in their saccadic-manual tasks, no ICE was observed in the present experiment, regardless of the CTOA. Note that, in Taylor and Klein's study, central arrow cues and peripheral onset cues were intermixed within blocks of trials. It is possible that this unique manipulation may have created an attentional set that gave rise to the ICEs observed in their saccadic-manual condition.

To closely replicate Taylor and Klein's (2000) design, we also used a visual cue-back signal to encourage the participants to orient attention back to the central fixation. In the saccadic-manual task, this cue-back signal stimulated the retinal locus corresponding to the uncued box, and thus may have invoked inhibition there (Wang et al., 2012). Although it is unclear whether this retinal stimulation could delay responses toward the uncued box, we eliminated this potential methodological confound in subsequent experiments.

\section{Experiment 2: no cue-back signal}

Experiment 2 was similar to Experiment 1, except that the visual cue-back signal was eliminated.

\section{Method}

Participants Twenty-two volunteers (14 females, eight males; mean age: 22.42 years) participated in Experiment 2. 
Apparatus, task procedure, and design The apparatus, task procedure, and design were identical to those of Experiment 1, except that the visual cue-back signal was eliminated and, in the saccadic-manual task, the participants were instructed to quickly saccade back to the central box, immediately after the eyes had reached the peripheral box indicated by the cue. As in Experiment 1, any incorrect eye movements or responses resulted in trial termination and recycling.

\section{Results}

Target RTs The RTs from successfully completed trials were cleansed using the same protocol as in Experiment 1. After data cleaning, $97.80 \%$ and $96.86 \%$ of the trials remained in the manual-manual and saccadicmanual tasks, respectively.

The mean target RTs are presented in Table 1. A repeated measures ANOVA revealed significant main effects for task $[F(1,21)=52.49, M S E=2,964, p<$ $\left..001, \eta_{G}{ }^{2}=.29\right]$ and CTOA $[F(1,21)=82.43, M S E=$ $\left.518, p<.001, \eta_{G}{ }^{2}=.1\right]$. RTs were generally longer in the saccadic-manual task, and were longer for the shortCTOA conditions. The main effect of cueing was marginally significant $[F(1,21)=3.15, M S E=728.2, p=$ $\left..09, \eta_{G}^{2}=.01\right]$. Significant two-way interactions occurred between cueing and CTOA $[F(1,21)=8.71$, $\left.M S E=211.3, p<.01, \eta_{G}^{2}=.005\right]$, due to a larger ICE in the longer-CTOA condition, and between task and CTOA $[F(1,21)=24.65, M S E=748, p<.001$, $\left.\eta_{G}{ }^{2}=.05\right]$, because the speeding up of RTs at the long CTOA was greater for the saccadic-manual task. The two-way interaction between cueing and task was not significant $[F(1,21)=0.24, M S E=263.24, p=.63$, $\left.\eta_{G}{ }^{2}=.00\right]$, whereas the three-way interaction was $[F(1$, 21) $\left.=8.16, M S E=229.3, p<.01, \eta_{G}{ }^{2}=.004\right]$. Planned comparisons revealed an ICE $(21 \mathrm{~ms})$ for the manualmanual task when the CTOA was $2,000 \mathrm{~ms}[t(21)=$ $4.16, p<.001]$. The ICEs in all other conditions did not reach significance [all $t \mathrm{~s}<0.96$, all $p \mathrm{~s}>.35$ ] (see Fig. 2b).

Target response errors Response error rates are also presented in Table 1. A repeated measures ANOVA revealed a significant main effect of cueing $[F(1,21)=$ 6.33, $\left.M S E=8.10, p=.02, \eta_{G}{ }^{2}=.02\right]$, with more errors occurring in uncued conditions. A significant two-way interaction between task and CTOA was also observed $\left[F(1,21)=8.83, M S E=3, p<.01, \eta_{G}{ }^{2}=.03\right]$, because the error rate decreased as the CTOA increased only in the saccadic-manual task. All other effects did not reach significance [all $F \mathrm{~s}<1.31$, all $p \mathrm{~s}>.26$ ].

\section{Discussion}

The results of Experiment 2 were consistent with those of Experiment 1 (see Fig. $2 \mathrm{a}$ and $\mathrm{b}$ ). ${ }^{1}$ In the manual-manual task, an ICE was observed only at the long CTOA; in the saccadic-manual task, no ICE was observed at either CTOA. Our failure to observe an ICE in the saccadicmanual task was in drastic contrast to previous observations of ICEs in similar tasks (e.g., Posner et al., 1985; Taylor \& Klein, 2000). However, since previous work has demonstrated the importance of the cue-back signal in revealing ICEs (e.g., Pratt \& Fischer, 2002; Prime \& Jolicœur, 2009; but see Possamaï, 1991), it is possible that we failed to observe ICEs in the saccadic-manual task because Experiment 2 did not include cue-back signals. This possibility was explored in Experiment 3 through the use of an auditory cue-back signal. With auditory cue-back signals, Satel and Wang (2012) observed robust ICE in a similar experimental setup.

\section{Experiment 3: auditory cue-back signal}

Experiment 3 followed the same design as Experiments 1 and 2, except that an auditory cue-back signal was used to reorient attention to the central fixation following responses to the cues.

\section{Method}

Participants A total of 16 participants (15 females, one male; mean age: 21.2 years) took part in Experiment 3.

Apparatus, task procedure, and design The apparatus, stimuli, and task procedure were the same as in Experiment 1, except that (a) the visual cue-back signal was replaced by an auditory beep, and (b) the short CTOA was extended to 1,200 ms, so as to allocate enough time for participants to respond to the cue-back signal.

\section{Results}

Target RTs After data cleansing, $97.70 \%$ and $97.13 \%$ of the successfully completed trials remained in the manualmanual and saccadic-manual tasks, respectively.

The mean target RTs in all conditions are presented in Table 1. An ANOVA of the RTs revealed significant main

\footnotetext{
${ }^{1}$ An ANOVA on the RTs, with Experiment (Exp. 1 vs. 2) as a factor, revealed a significant three-way interaction between cueing, CTOA, and task $\left[F(1,42)=9.98, p<.01, \eta_{\mathrm{G}}{ }^{2}=.002\right]$. The four-way interaction involving experiment did not reach significance $[F(1,42)=1.97, p=$ $\left..17, \eta_{\mathrm{G}}^{2}=.0004\right]$.
} 
effects for task $\left[F(1,15)=75.68, M S E=2,472, p<.001, \eta_{G}{ }^{2}\right.$ $=.42], \mathrm{CTOA}\left[F(1,15)=21.82, M S E=985, p<.001, \eta_{G}{ }^{2}=\right.$ $.08]$, and cueing $\left[F(1,15)=5.16, M S E=1,353, p<.05, \eta_{G}{ }^{2}=\right.$ $.03]$. The main effect of cueing emerged because RTs were generally longer for cued than for uncued targets, suggesting the observation of an overall ICE. The main effects of task and CTOA were due to faster responses in the manual-manual condition and at the long CTOA. A significant two-way interaction was observed between CTOA and task $[F(1,15)=$ $\left.24.47, M S E=514, p<.001, \eta_{G}{ }^{2}=.05\right]$, because there was a greater reduction in RTs at the longer CTOA in the saccadicmanual task. The two-way interactions between cueing and CTOA $\left[F(1,15)=3.25, M S E=247.2, p=.09, \eta_{G}{ }^{2}=.003\right]$ and cueing and task $\left[F(1,15)=3.31, M S E=227.1, p=.09, \eta_{G}{ }^{2}=\right.$ .003] approached significance. The three-way interaction between task, cueing, and CTOA did not reach significance $\left[F(1,15)=2.87, M S E=372.8, p=.11, \eta_{G}{ }^{2}=.004\right]$. Planned comparisons revealed that, consistent with Experiments 1 and 2, in the manual-manual task an ICE (21 ms) was observed only at the long CTOA (2,000 ms) $[t(15)=2.39, p<.05]$. However, with auditory cue-back signals, ICEs emerged in the saccadic-manual task at both short $(21 \mathrm{~ms})[t(15)=2.23, p<.05]$ and long $(19 \mathrm{~ms})[t(15)=2.20$, $p=.05]$ CTOAs.

Target response errors Response error rates are also presented in Table 1. A repeated measures ANOVA revealed marginal main effects for task $[F(1,15)=4.40, M S E=14.01, p=.05$, $\left.\eta_{G}{ }^{2}=.03\right]$, and cueing $[F(1,15)=4.34, M S E=7.39, p=.05$, $\left.\eta_{G}{ }^{2}=.01\right]$. Error rates were higher in the manual-manual task and in cued conditions. A marginal two-way interaction was observed between task and cueing $[F(1,15)=3.97, M S E=$ 21.93, $\left.p=.06, \eta_{G}{ }^{2}=.04\right]$, because the effect of cueing was more prominent in the manual-manual task. The three-way interaction also reached marginal significance $[F(1,15)=$ $\left.3.84, M S E=12.51, p=.07, \eta_{G}{ }^{2}=.02\right]$, because the error rate increased with CTOA in the manual-manual task, whereas it decreased with CTOA in the saccadic-manual task. All other effects did not reach significance [all $F_{\mathrm{S}}<2.06$, all $p \mathrm{~s}>.17$ ].

\section{Discussion}

Experiment 3 produced two important findings. First, with an auditory cue-back signal, reliable ICEs were observed in the saccadic-manual task. This was in contrast with the two previous experiments, in which no ICE was observed with a visual cue back (Exp. 1) or without any cue back (Exp. 2). It thus seems that, although making a saccadic response to a cue is sufficient to evoke an ICE (e.g., Posner et al., 1985; Rafal et al., 1989; Satel \& Wang, 2012; Taylor \& Klein, 2000), the ICE will only delay manual responses under certain conditions. Second, a reliable ICE was again observed only at the long CTOA in the manual-manual task.

\section{Experiment 4: time course}

The findings of Experiments 1-3 clearly show that (a) ICEs can be evoked and revealed with arrow cues/targets and manual localization responses, although only with sufficiently long time intervals between the cue and target, and (b) ICEs evoked by saccadic responses to arrow cues do carry over to the skeletomotor system to delay manual responses. To further understand these ICEs, it is necessary to characterize their time courses.

\section{Method}

Participants Seventeen volunteers participated in Experiment 4. One of them was excluded from the analysis because she did not finish the tasks. The mean age of the remaining 16 participants (13 females, three males) was 19.38 years.

Apparatus, task procedure, and design The apparatus, task procedure, and design were the same as in Experiment 3, except that three CTOAs $(1,200,2,000$, and 3,000 ms), rather than two, were tested.

\section{Results}

Target RTs After cleansing, $97.67 \%$ and $97.17 \%$ of the successfully completed trials remained in the manual-manual and saccadic-manual tasks, respectively.

The mean target RTs in all conditions are presented in Table 1. A repeated measures ANOVA on the RTs revealed significant main effects for task $[F(1,15)=52.44, M S E=1$, $\left.554, p<.001, \eta_{G}{ }^{2}=.17\right]$, CTOA $[F(2,30)=40.49, M S E=$ $\left.687, p<.001, \eta_{G}{ }^{2}=.12\right]$, and cueing $[F(1,15)=14.36, M S E=$ $\left.1,187, p<.001, \eta_{G}{ }^{2}=.04\right]$. The RTs were generally longer in the saccadic-manual task and for cued targets, and decreased as the CTOA increased. A significant two-way interaction was observed between task and CTOA $[F(2,30)=23.91, M S E=$ $710, p<.001, \eta_{G}^{2}=.08$ ], due to a greater reduction in RTs as CTOA increased in the saccadic-manual task. The two-way interactions between cueing and CTOA $[F(1,15)=0.03, M S E$ $\left.=123.52, p=.97, \eta_{G}{ }^{2}=.00\right]$ and cueing and task $[F(1,15)=$ $\left.0.49, M S E=285, p=.49, \eta_{G}{ }^{2}=.00\right]$ were not significant. The three-way interaction between task, CTOA, and cueing was significant $\left[F(2,30)=6.15, M S E=218.5, p<.01, \eta_{G}{ }^{2}=\right.$ .006], because the ICEs increased with CTOA in the manual-manual task, whereas they decreased in the saccadic-manual task (see Fig. 2d). Planned comparisons revealed that, in the manual-manual task, ICEs were observed for the 3,000$\mathrm{ms}(26 \mathrm{~ms})[t(15)=4.35, p<.01]$ and $2,000-\mathrm{ms}(16 \mathrm{~ms})[t(15)$ $=2.54, p<.05]$ CTOAs, but not for the 1,200-ms CTOA $[t(15)=1.28, p=.22]$. In the saccadic-manual task, ICEs were observed for the 1,200-ms $(29 \mathrm{~ms})[t(15)=6.71, p<.001]$ and 
$2,000-\mathrm{ms}(22 \mathrm{~ms})[t(15)=3.25, p<.01]$ CTOAs, but not for the $3,000-\mathrm{ms}$ CTOA $[t(15)=1.16, p=.26]$.

As is clear from Fig. 2d, the ICE generally decreased as the CTOA increased in the saccadic-manual task, much like the ICEs observed in the classic cueing paradigm (e.g., Fecteau \& Munoz, 2005; Samuel \& Kat, 2003). Confirming this trend, an ANOVA on the ICEs in the saccadic-manual task revealed a significant main effect of CTOA $[F(2,30)=4.15, M S E=$ $\left.354.4, p<.05, \eta_{G}{ }^{2}=.03\right]$. Pairwise comparisons revealed a significant difference in ICEs between the 1,200-ms and 3, 000 -ms CTOAs $[t(15)=2.29, p<.05]$, and a marginally significant difference between the 2,000-ms and 3,000-ms CTOAs $[t(15)=2.09, p=.05]$; the difference between the 1 , 200-ms and 2,000-ms CTOAs did not reach significance $[t(15)=1.25, p=.22]$. The manual-manual task, however, produced an ICE that started later and had no sign of decreasing at the longest CTOA tested in the present experiment (3, $000 \mathrm{~ms})$. An ANOVA of the ICEs in this task also revealed a significant effect of CTOA $[F(2,30)=3.72, M S E=329.7, p<$ $\left..05, \eta_{G}^{2}=.04\right]$. Pairwise comparisons revealed only a significant difference in ICE between the 1,200- and 3,000-ms CTOAs $[t(15)=2.71, p<.05]$; the differences between the 1,200- and 2,000-ms CTOAs $[t(15)=1.28, p=.22]$ and the 2 , 000 - and 3,000-ms CTOAs $[t(15)=1.44, p=.17]$ did not reach significance.

Target response errors Response error rates are presented in Table 1. An ANOVA revealed only a significant two-way interaction between task and CTOA $[F(2,30)=3.49$, MSE $\left.=8.70, p<.05, \eta_{G}{ }^{2}=.02\right]$. Again, error rates appeared to increase with CTOA in the manual-manual task, but to decrease with CTOA in the saccadic-manual task. All other effects did not reach significance $\left[F_{\mathrm{S}}<2.07, p_{\mathrm{s}}>.14\right]$.

\section{Discussion}

In Experiment 4, we examined the time courses of ICEs evoked by saccadic and manual responses. As is shown in Fig. 2d, the ICE in the saccadic-manual task generally decreased as the CTOA increased, whereas that in the manualmanual task had a trend to increase with CTOA. It is unclear why this pattern of results emerged, but it is likely that the ICE in the manual-manual tasks originates from neurodynamic mechanisms that are distinct from the oculomotor IOR effects evoked by saccadic cue responses.

\section{General discussion}

With two cueing tasks, in the present study we examined output-based ICEs evoked by saccadic and manual localization responses to arrow cues. ICEs were consistently evoked and revealed with manual responses to arrow cues and targets in all experiments - but only when the CTOA was 2,000 ms or longer. Following saccadic responses to arrow cues, ICEs were also observed, but only when an auditory cue-back signal was supplied to reorient the participant's gaze to the central fixation (Exps. 3-4). Furthermore, the present experiments show that the magnitudes of the ICEs evoked by saccadic responses generally decreased as the CTOA increased, mimicking the pattern of ICEs observed with peripheral onset cues (Samuel \& Kat, 2003). The ICEs evoked by manual responses to the cue, however, appeared later in time and did not decrease even after $3 \mathrm{~s}$ had elapsed since cue onset (Exp. 4).

In the present experiments, the cues and targets were both arrows at fixation. One might suggest that the RT cost observed for targets pointing in the same direction as the cue might well be a shape-based, nonspatial repetition disadvantage (Fox \& de Fockert, 2001; Riggio, Patteri, \& Umiltà, 2003). We believe that this is unlikely for several reasons. First, the visual and auditory cue-back signals could have functioned as the "neutral attractors" that are critical for nonspatial repetition disadvantages (Fox \& de Fockert, 2001; Law, Pratt, \& Abrams, 1995; Taylor \& Klein, 1998). However, ICEs were not always observed when these neutral attractors were present (Exps. 1, 3, and 4). Second, the ICEs observed in the present experiments cannot be attributed solely to a repetition disadvantage, because arrows can reflexively orient attention (e.g., Hommel, Pratt, Colzato, \& Godijn, 2001; Stevens, West, Al-Aidroos, Weger, \& Pratt, 2008). Third, and most importantly, in Fox and de Fockert (2001) and other studies of nonspatial ICEs (e.g., Hu \& Samuel, 2011; Kwak \& Egeth, 1992; Law et al., 1995; Taylor \& Klein, 1998) the required detection or discrimination responses entailed no spatial information, whereas in the present work, the arrow cues and targets both required spatial localization responses. Thus, the ICEs reported here cannot be regarded as nonspatial.

The most important finding of the present study was that a robust ICE was observed when simple manual localization responses were made to central arrow cues. This ICE may arise from habituation of overt orienting responses (Dukewich, 2009), or it may be "merely an epiphenomenon arising from neural adaptation within motor control networks" (Cowper-Smith et al., 2013). Regardless of the underlying mechanism(s), the available evidence seems to suggest that this ICE is linked to skeletomotor activation. This ICE does not depend on the type of manual response required - it can be evoked and revealed with either simple buttonpresses (present experiments) or reaching responses (Cowper-Smith et al., 2013). Also, and more importantly, this ICE takes a longer time to emerge on a behavioral level than does "traditional IOR," and its magnitude does not seem to decrease even after $3 \mathrm{~s}$ have elapsed since cue onset. This unique time course is in dramatic contrast to those of other ICEs that have been reported in the literature (Klein, 2000, 2004; Samuel \& Kat, 2003; 
Wang et al., 2012). It is worth noting that the ICE evoked by oculomotor activation, as revealed by the saccadic-manual task, is largely dispersed $3 \mathrm{~s}$ after cue onset. These observations dovetail with the finding that, in visual search tasks, IOR (or inhibitory) tags at manually searched locations (Thomas et al., 2006) last longer than those at previously fixated locations (Dodd, Van der Stigchel, \& Hollingworth, 2009), implying that the oculomotor and skeletomotor systems are responsible for relatively fast and slow overt orienting, respectively.

Since the discovery of IOR, various ICEs have been shown to be closely tied to the oculomotor system (e.g., Dorris et al., 2002; Posner et al., 1985; Sapir, Soroker, Berger, \& Henik, 1999; Satel et al., 2011; Wang, Satel, Trappenberg, \& Klein, 2011). The term "oculomotor IOR" has been used in several recent studies to stress the importance of oculomotor activation in the generation of ICEs (or IOR; e.g., Hilchey et al., 2014; Klein \& Hilchey, 2011; Wang et al., 2012). In the present experiment, we explored whether oculomotor ICEs, when evoked by saccadic responses to central arrow cues, carry over to the skeletomotor system to delay manual responses. No ICE was observed when a visual (Exp. 1) or no (Exp. 2) cue-back signal was used in the saccadic-manual task. When an auditory cue-back signal was used in Experiments 3 and 4, however, ICEs were observed in the saccadic-manual task at both short and long CTOAs. This pattern of results suggests that auditory cue-back signals are critical for oculomotor ICEs to transfer to the skeletomotor system. ${ }^{2}$ However, given the methodological differences across experiments, this finding should not be overstated.

\section{Conclusions}

In the present study, we examined the output-based inhibitory cueing effects evoked by saccadic and manual localization responses to arrow cues. We showed that ICEs evoked by manual and saccadic responses to central arrow cues can both be revealed with manual target responses, and that the ICE evoked by manual cue responses has a unique time course. We conclude that ICEs linked to skeletomotor activation do exist and that oculomotor ICEs can carry over to the skeletomotor system to delay manual responses.

\footnotetext{
2 This supposition was supported by an ANOVA of the RTs from the saccadic-manual tasks. Note that only the 1,200-ms and 2,000-ms CTOAs of Experiment 4 were considered, and that Experiments 1 and 2 were regarded as conditions without an auditory cue back. The results revealed a significant effect of cueing (cued vs. uncued) $[F(1,62)=21.65$, $\left.M S E=622, p<.001, \eta_{G}{ }^{2}=.02\right]$ that interacted with the cue-back signal (with vs. without auditory cue back) $[F(1,62)=6.85, M S E=622, p<.05$, $\left.\eta_{G}{ }^{2}=.01\right]$. The three-way interaction involving CTOA (short vs. long) did not reach significance $\left[F(1,62)=0.63, M S E=228.71, p=.43, \eta_{G}{ }^{2}=\right.$ $.00]$. The two-way interaction between cueing and cue-back signal suggests that the auditory cue back did play a role in the saccadic-manual task, which assessed whether oculomtor ICEs carry over to the skeletomotor system.
}

Author note This project was supported by grants from the National Natural Science Foundation of China (Grant No. 31371133) and the Zhejiang Provincial Natural Science Foundation (Grant No. LY13C090007) to Z.W.

\section{References}

Abrams, R. A., \& Dobkin, R. S. (1994). Inhibition of return: Effects of attentional cueing on eye movement latencies. Journal of Experimental Psychology: Human Perception and Performance, 20, 467-477. doi:10.1037/0096-1523.20.3.467

Bakeman, R. (2005). Recommended effect size statistics for repeated measures designs. Behavior Research Methods, 37, 379-384. doi: 10.3758/BF03192707

Berlucchi, G., Di Stefano, M., Marzi, C. A., Morelli, M., \& Tassinari, G. (1981). Direction of attention in the visual field as measured by a reaction time paradigm. Behavioural Brain Research, 2, 244-245. doi:10.1016/0166-4328(81)90066-8

Briand, K. A., Larrison, A. L., \& Sereno, A. B. (2000). Inhibition of return in manual and saccadic response systems. Perception \& Psychophysics, 62, 1512-1524.

Chica, A. B., Taylor, T. L., Lupiáñez, J., \& Klein, R. M. (2010). Two mechanisms underlying inhibition of return. Experimental Brain Research, 201, 25-35. doi:10.1007/s00221-009-2004-1

Clohessy, A. B., Posner, M. I., Rothbart, M. K., \& Vecera, S. P. (1991). The development of inhibition of return in early infancy. Journal of Cognitive Neuroscience, 3, 345-350. doi:10.1162/jocn.1991.3.4.345

Cohen, Y. (1981). Internal and external control of orienting (Unpublished doctoral dissertation). University of Oregon, Eugene, OR.

Cowper-Smith, C. D., Eskes, G. A., \& Westwood, D. A. (2013). Motor inhibition of return can affect prepared reaching movements. Neuroscience Letters, 541, 83-86. doi:10.1016/j.neulet.2013.02.033

Dodd, M. D., Van der Stigchel, S., \& Hollingworth, A. (2009). Novelty is not always the best policy: Inhibition of return and facilitation of return as a function of visual task. Psychological Science, 20, 333339. doi:10.1111/j.1467-9280.2009.02294.x

Dorris, M. C., Klein, R. M., Everling, S., \& Munoz, D. P. (2002). Contribution of the primate superior colliculus to inhibition of return. Journal of Cognitive Neuroscience, 14, 1256-1263. doi:10. 1162/089892902760807249

Dukewich, K. R. (2009). Reconceptualizing inhibition of return as habituation of the orienting response. Psychonomic Bulletin \& Review, 16, 238-251. doi:10.3758/PBR.16.2.238

Fecteau, J. H., \& Munoz, D. P. (2005). Correlates of capture of attention and inhibition of return across stages of visual processing. Journal of Cognitive Neuroscience, 17, 1714-1727. doi:10.1162/ 089892905774589235

Fischer, M. H., Pratt, J., \& Neggers, S. F. W. (2003). Inhibition of return and manual pointing movements. Perception \& Psychophysics, 65, 379-387. doi:10.3758/BF03194569

Fox, E., \& de Fockert, J.-W. (2001). Inhibitory effects of repeating color and shape: Inhibition of return or repetition blindness? Journal of Experimental Psychology: Human Perception and Performance, 27, 798-812. doi:10.1037/0096-1523.27.4.798

Hilchey, M. D., Klein, R. M., \& Satel, J. (2014). Returning to "inhibition of return" by dissociating long-term oculomotor IOR from shortterm sensory adaptation and other nonoculomotor "inhibitory" cueing effects. Journal of Experimental Psychology: Human Perception and Performance, 40, 1603-1616. doi:10.1037/ a0036859

Hommel, B., Pratt, J., Colzato, L., \& Godijn, R. (2001). Symbolic control of visual attention. Psychological Science, 12, 360-365. 
Hu, F. K., \& Samuel, A. G. (2011). Facilitation versus inhibition in nonspatial attribute discrimination tasks. Attention, Perception, \& Psychophysics, 73, 784-796. doi:10.3758/s13414-010-0061-y

Ivanoff, J., \& Klein, R. M. (2001). The presence of a nonresponding effector increases inhibition of return. Psychonomic Bulletin \& Review, 8, 307-314. doi:10.3758/BF03196166

Ivanoff, J., Klein, R. M., \& Lupiáñez, J. (2002). Inhibition of return interacts with the Simon effect: An omnibus analysis and its implications. Perception \& Psychophysics, 64, 318-327. doi:10.3758/ BF03195794

Jonides, J. (1981). Voluntary versus automatic control over the mind's eye's movement. In J. Long \& A. Baddeley (Eds.), Attention and performance IX (pp. 187-203). Hillsdale, NJ: Erlbaum.

Khatoon, S., Briand, K. A., \& Sereno, A. B. (2002). The role of response in spatial attention: Direct versus indirect stimulus-response mappings. Vision Research, 42, 2693-2708. doi:10.1016/S00426989(02)00327-9

Klein, R. M. (2000). Inhibition of return. Trends in Cognitive Sciences, 4, 138-147. doi:10.1016/S1364-6613(00)01452-2

Klein, R. M. (2004). Orienting and inhibition of return. In M. S. Gazzaniga (Ed.), The cognitive neurosciences (3rd ed., pp. 545559). Cambridge, MA: MIT Press.

Klein, R. M., \& Hilchey, M. D. (2011). Oculomotor inhibition of return. In S. Liversedge, I. D. Gilchrist, \& S. Everling (Eds.), The Oxford handbook of eye movements (pp. 471-492). Oxford, UK: Oxford University Press. doi:10.1093/oxfordhb/9780199539789.013.0026

Klein, R. M., Schmidt, W. C., \& Müller, H. J. (1998). Disinhibition of return: Unnecessary and unlikely. Perception \& Psychophysics, 60, 862-872. doi:10.3758/BF03206069

Kwak, H.-W., \& Egeth, H. (1992). Consequences of allocating attention to locations and to other attributes. Perception \& Psychophysics, 51, 455-464. doi:10.3758/BF03211641

Law, M. B., Pratt, J., \& Abrams, R. A. (1995). Color-based inhibition of return. Perception \& Psychophysics, 57, 402-408. doi:10.3758/ BF03213064

Lupiáñez, J., Milán, E. G., Tornay, F. J., Madrid, E., \& Tudela, P. (1997). Does IOR occur in discrimination tasks? Yes, it does, but later. Perception \& Psychophysics, 59, 1241-1254. doi:10.3758/ BF03214211

Maylor, E. A. (1985). Facilitatory and inhibitory components of orienting in visual space. In M. I. Posner \& O. S. M. Marin (Eds.), Attention and performance XI: Mechanism of attention (pp. 189-204). Hillsdale, NJ: Erlbaum.

Müller, N. G., \& Kleinschmidt, A. (2007). Temporal dynamics of the attentional spotlight: Neuronal correlates of attentional capture and inhibition of return in early visual cortex. Journal of Cognitive Neuroscience, 19, 587-593. doi:10.1162/jocn.2007.19.4.587

Posner, M. I., \& Cohen, Y. (1984). Components of visual orienting. In H. Bouma \& D. G. Bouwhuis (Eds.), Attention and performance X: Control of language processes (Vol. 10, pp. 531-556). Hillsdale, NJ: Erlbaum.

Posner, M. I., Rafal, R. D., Choate, L. S., \& Vaughan, J. (1985). Inhibition of return: Neural basis and function. Cognitive Neuropsychology, 2, 211-228. doi:10.1080/02643298508252866

Possamaï, C. A. (1991). A responding hand effect in a simple-RT precueing experiment: Evidence for a late locus of facilitation. Acta Psychologica, 77, 47-63.

Pratt, J., \& Fischer, M. H. (2002). Examining the role of the fixation cue in inhibition of return. Canadian Journal of Experimental Psychology, 56, 294-301. doi:10.1037/h0087405

Prime, D. J., \& Jolicœur, P. (2009). On the relationship between occipital cortex activity and inhibition of return. Psychophysiology, 46, 1278 1287. doi:10.1111/j.1469-8986.2009.00858.x

R Development Core Team. (2015). R: A language and environment for statistical computing. Vienna, Austria: R Foundation for Statistical Computing. Retrieved from www.R-project.org
Rafal, R. D., Calabresi, P. A., Brennan, C. W., \& Sciolto, T. K. (1989). Saccade preparation inhibits reorienting to recently attended locations. Journal of Experimental Psychology: Human Perception and Performance, 15, 673-685. doi:10. 1037/0096-1523.15.4.673

Riggio, L., Patteri, I., \& Umiltà, C. (2003). Location and shape in inhibition of return. Psychological Research, 68, 41-54. doi:10.1007/ s00426-003-0136-7

Samuel, A. G., \& Kat, D. (2003). Inhibition of return: A graphical metaanalysis of its time course and an empirical test of its temporal and spatial properties. Psychonomic Bulletin \& Review, 10, 897-906. doi:10.3758/BF03196550

Sapir, A., Soroker, N., Berger, A., \& Henik, A. (1999). Inhibition of return in spatial attention: Direct evidence for collicular generation. Nature Neuroscience, 2, 1053-1054. doi:10.1038/15977

Satel, J., Hilchey, M. D., Wang, Z., Story, R., \& Klein, R. M. (2013). The effects of ignored versus foveated cues upon inhibition of return: An event-related potential study. Attention, Perception, \& Psychophysics, 75, 29-40. doi:10.3758/s13414-012-0381-1

Satel, J., \& Wang, Z. (2012). Investigating a two causes theory of inhibition of return. Experimental Brain Research, 223, 469-478. doi:10. 1007/s00221-012-3274-6

Satel, J., Wang, Z., Trappenberg, T. P., \& Klein, R. M. (2011). Modeling inhibition of return as short-term depression of early sensory input to the superior colliculus. Vision Research, 51, 987-996. doi:10.1016/j. visres.2011.02.013

Stevens, S. A., West, G. L., Al-Aidroos, N., Weger, U. W., \& Pratt, J. (2008). Testing whether gaze cues and arrow cues produce reflexive or volitional shifts of attention. Psychonomic Bulletin \& Review, 15, 1148-1153. doi:10.3758/PBR.15.6.1148

Sumner, P. (2006). Inhibition versus attentional momentum in cortical and collicular mechanisms of IOR. Cognitive Neuropsychology, 23, 1035-1048.

Sumner, P., Nachev, P., Vora, N., Husain, M., \& Kennard, C. (2004). Distinct cortical and collicular mechanisms of inhibition of return revealed with S cone stimuli. Current Biology, 14, 2259-2263. doi: 10.1016/j.cub.2004.12.021

Taylor, T. L., \& Klein, R. M. (1998). Inhibition of return to color: A replication and nonextension of Law, Pratt, and Abrams (1995). Perception \& Psychophysics, 60, 1452-1456. doi:10.3758/ BF03208005

Taylor, T. L., \& Klein, R. M. (2000). Visual and motor effects in inhibition of return. Journal of Experimental Psychology: Human Perception and Performance, 26, 1639-1656. doi:10.1037/00961523.26.5.1639

Thomas, L. E., Ambinder, M. S., Hsieh, B., Levinthal, B., Crowell, J. A., Irwin, D. E., . . . Wang, R. F. (2006). Fruitful visual search: Inhibition of return in a virtual foraging task. Psychonomic Bulletin \& Review, 13, 891-895. doi:10.3758/BF03194015

Van Selst, M., \& Jolicour, P. (1994). A solution to the effect of sample size on outlier elimination. Quarterly Journal of Experimental Psychology, 47A, 631-650. doi:10.1080/14640749408401131

Wang, Z., Satel, J., Trappenberg, T. P., \& Klein, R. M. (2011). Aftereffects of saccades explored in a dynamic neural field model of the superior colliculus. Journal of Eye Movement Research, 4, 1-16.

Wang, Z., Satel, J., \& Klein, R. M. (2012). Sensory and motor mechanisms of oculomotor inhibition of return. Experimental Brain Research, 218, 441-453. doi:10.1007/s00221-012-3033-8

Werner, W. (1993). Neurons in the primate superior colliculus are active before and during arm movements to visual targets. European Journal of Neuroscience, 5, 335-340. doi:10.1111/j.1460-9568. 1993.tb00501.x

Zhang, Y., \& Zhang, M. (2011). Spatial working memory load impairs manual but not saccadic inhibition of return. Vision Research, 51, 147-153. doi:10.1016/j.visres.2010.10.022 